\title{
Le voir et le dire. Images et discours politiques dans l'editorial cartoon aux États-Unis
}

\author{
Donna Andréolle
}

\section{(2) OpenEdition \\ Journals}

\section{Édition électronique}

URL : http://journals.openedition.org/asp/2270

DOI : $10.4000 /$ asp. 2270

ISBN : 978-2-8218-0382-4

ISSN : 2108-6354

Éditeur

Groupe d'étude et de recherche en anglais de spécialité

\section{Édition imprimée}

Date de publication : 31 décembre 2000

Pagination : $327-348$

ISSN : 1246-8185

\section{Référence électronique}

Donna Andréolle, « Le voir et le dire. Images et discours politiques dans l'editorial cartoon aux États-

Unis », ASp [En ligne], 27-30 | 2000, mis en ligne le 26 février 2011, consulté le 30 avril 2019. URL

http://journals.openedition.org/asp/2270 ; DOI : 10.4000/asp.2270

Ce document a été généré automatiquement le 30 avril 2019.

Tous droits réservés 


\title{
Le voir et le dire. Images et discours politiques dans l'editorial cartoon aux États-Unis
}

\author{
Donna Andréolle
}

1 Voici deux phrases connues qui résument fort bien la fonction, et le fonctionnement, de l' editorial cartoon. Combinaison d'un dessin humoristique ou caricatural et de propos soit sous forme de légende, soit sous forme de bulles qui font parler les personnages, l'editorial cartoon prend son appui dans différentes stratégies de subversion que nous tenterons d'analyser dans le présent article. Nous chercherons à démontrer en quoi ces dessins sont aussi bien un moyen privilégié de commentaire éditorial, qu'une forme spécialisée de discours politique et politisé.

2 Tout d'abord, cette technique particulière de commentaire requiert la maitrise de multiples compétences :

- hormis les qualités purement artistiques nécessaires à la réalisation d'un dessin de ce type, suivant le journal pour lequel il travaille, l'artiste doit posséder une connaissance approfondie de l'actualité locale, régionale, nationale et/ou internationale ;

- il doit pouvoir faire appel à de nombreuses références culturelles, qu'elles soient de nature historique, politique, littéraire, cinématographique, ou télévisuelle, pour ne nommer que les plus évidentes ;

- enfin, l'artiste doit être capable avant tout de pérenniser l'éphémère, c'est-à-dire de saisir un fait significatif du «maintenant » politique et d'en faire une réalisation artistique qui perdurera.

3 À titre d'exemple, on peut voir dans le dessin 1 (cartoon 1) la superposition d'un fait divers politique et d'une situation politique d'envergure : lors d'une conférence de presse en mai 1966, en réponse aux questions concernant son état de santé après une opération de la vésicule biliaire, le Président Johnson a soulevé sa chemise pour montrer sa cicatrice; le lendemain, la photo de Johnson exposant son ventre aux journalistes a fait la une des grands quotidiens nationaux. Ici donc l'artiste David Levine a profité de cet «événement » politique pour dénoncer un autre fait politique bien plus grave : l'escalade 
des interventions militaires en Asie du Sud-est dont Johnson était responsable, commentaire réussi en transformant tout simplement la cicatrice désormais célèbre en une carte du Vietnam.

Cartoon 1

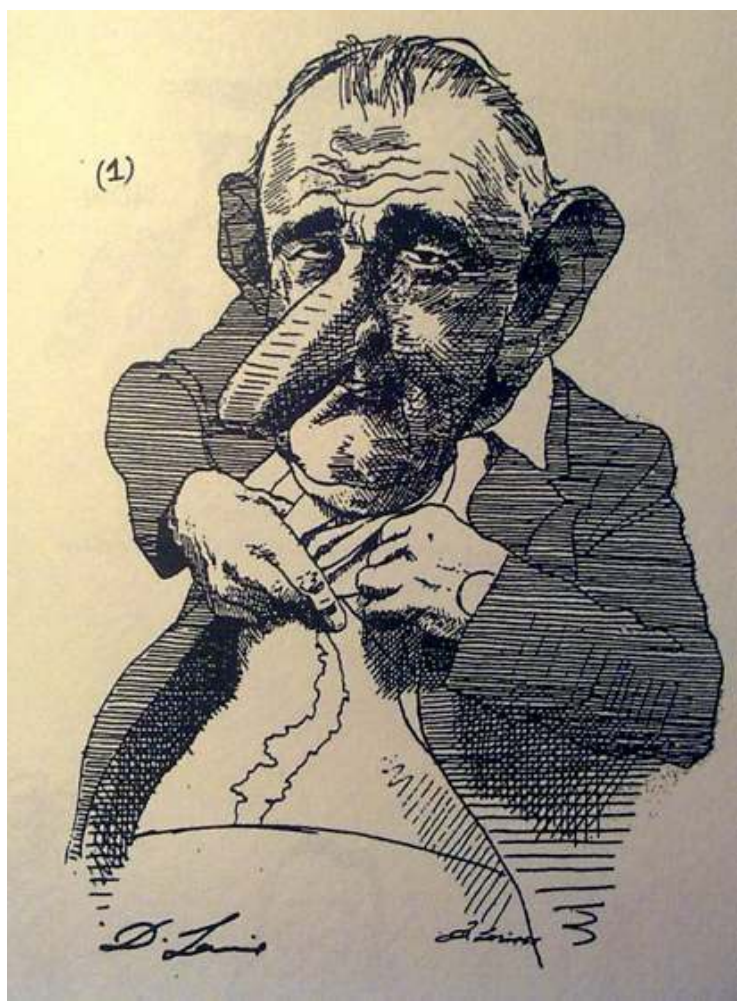

En même temps, l'artiste doit savoir éviter à tout prix les écueils de son art: la trop grande simplification ou la schématisation de l'événement visé qui risquerait de diminuer le commentaire politique désiré ; l'utilisation de métaphores surexploitées comme le naufrage du Titanic, le nez de Pinocchio qui s'allonge, la campagne présidentielle transformée en match de boxe...; ou encore la mise en œuvre de références culturelles trop obscures au risque de rendre le cartoon illisible pour le lecteur moyen. Globalement donc, l'artiste-spécialiste de l'editorial cartoon a pour tâche de discerner rapidement l'importance de telle ou telle actualité politique, et de choisir un angle d'approche ainsi qu'un style humoristique le mieux approprié au traitement du sujet, avant de trouver une représentation métaphorique originale et surtout rapidement accessible au plus grand nombre de lecteurs (voir Kallaugher, site Internet). Dans ce sens, les techniques de l' editorial cartoon ressemblent beaucoup à celles déployées dans la publicité: attirer l'attention, susciter l'intérêt, produire un effet de surprise qui débouche sur la compréhension des sens multiples de l'image et du texte afin d'obtenir l'adhésion du lecteur au point de vue exprimé ${ }^{1}$.

Historiquement, l'apparition aux États-Unis du dessin à caractère politique remonte au XVIII ${ }^{e}$ siècle, lorsque Benjamin Franklin publia, dans le Pennsylvania Gazette du 9 mai 1754 le dessin d'un serpent coupé en morceaux accompagné de la légende "JOIN, or DIE ». Premier editorial cartoon à être publié dans un journal américain, ce dessin exprimait le désir de Franklin de convaincre les colonies à rassembler leurs forces militaires dans la guerre contre les Français et les Indiens. La métaphore du serpent repose sur une 
croyance populaire selon laquelle l'assemblage des morceaux d'un serpent découpé, effectué avant le coucher de soleil, permettrait au reptile de revivre. Devenue alors le symbole américain du dicton « l'union fait la force », cette même image a été reprise dans la presse nationale lors de deux autres événements politiques de taille dans l'histoire des colonies, en 1765 (pour rallier les forces coloniales à la cause du soulèvement contre The Stamp Act) et à la veille de la Révolution américaine en 1774.

Il faut attendre néanmoins la deuxième moitié du XIX siècle avant de trouver l'art de l' editorial cartoon à son apogée, notamment grâce à l'œuvre de Thomas Nast, l'un des artistes les plus célèbres dans l'histoire de ce genre aux États-Unis. La célébrité de Thomas Nast et d'autres artistes comme Joseph Keppler coïncide avec la popularité, à cette période, de la presse illustrée telle que Harper's Weekly; mais Nast doit sa renommée avant tout à ses cartoons qui ont attiré l'attention du public sur la corruption politique de «Boss» Tweed, puissant leader du parti démocrate dans la ville de New York, dont l'affaire est connue sous le nom de Tammany Hall. Les attaques répétées de l'artiste dans la presse ont mené, à terme, à l'arrestation et à l'emprisonnement de Tweed; encore aujourd'hui Thomas Nast reste une référence de l'art éditorial non seulement parce qu'il avait démontré le pouvoir du cartoon, mais aussi parce qu'il a su perfectionner des stratégies de caricature au service d'un message politique ${ }^{2}$. Enfin, il faut mentionner une autre dimension importante contribuant à la popularité de l'editorial cartoon de cette période : la visualisation de faits politiques par le biais de l'image a rendu accessible le commentaire éditorial aux populations illettrées et immigrantes très nombreuses à la fin du siècle ; c'est donc à cette époque que l'editorial cartoon devient véritablement un pilier de la culture populaire.

Grâce à l'œuvre de Thomas Nast en particulier, l'efficacité de l'editorial cartoon en tant qu'arme médiatique redoutable n'est plus à prouver. L'arrivée des nouvelles technologies de l'information, offrant à l'artiste une médiatisation planétaire, n'a fait que renforcer la popularité et l'impact de cet art. Les grands artistes actuels aux États-Unis, Pat Oliphant, Jim Borgman, Jeff MacNelly (et d'autres), ont leurs propres sites sur lesquels on peut voir leur production du jour ou consulter des archives importantes; certains de ces sites comportent même l'explication détaillée du fait politique traité dans chaque cartoon (site de la Library of Congress dédié aux œuvres de Pat Oliphant) ou les réflexions de l'artiste sur le pourquoi de son choix d'événement (site de Scott Bateman)33. Il est également possible de consulter les editorial cartoons du jour sur le thème de la campagne présidentielle en cours sur les sites des grandes chaînes américaines comme CNN, ABC News et CBS News. C'est donc en examinant les œuvres de ces quelques artistes de grande envergure que nous allons analyser trois grandes stratégies déployées pour commenter l'actualité politique : le détournement de propos "médiatiques", le décalage entre sens propre et sens figuré (qu'il soit pictural ou lexical) et l'utilisation d'images ou de discours historiques pour illustrer un fait contemporain. Bien entendu, ces stratégies ne sont en aucun cas hermétiques ou mutuellement exclusives car c'est justement la mise en œuvre habile de plusieurs stratégies à la fois qui fait la richesse et la réussite de l'editorial cartoon.

Il convient, par ailleurs, de ne pas perdre de vue la très forte médiatisation du discours politique aux États-Unis, médiatisation développée depuis l'avènement de la télévision. On notera en passant que les stratèges de la campagne présidentielle de Dwight Eisenhower (en 1952) ont utilisé le nouvel outil de communication qu'était la télévision pour créer et tester l'impact de la publicité électorale télévisée. De nos jours il existe tout un lexique spécialisé pour décrire le traitement du discours politique, comme le terme 
sound bites qui désigne ces petites phrases proches du slogan publicitaire dont les candidats à la présidence truffent leurs discours dans l'espoir qu'elles soient citées dans le journal télévisé du soir.

Cartoon 2

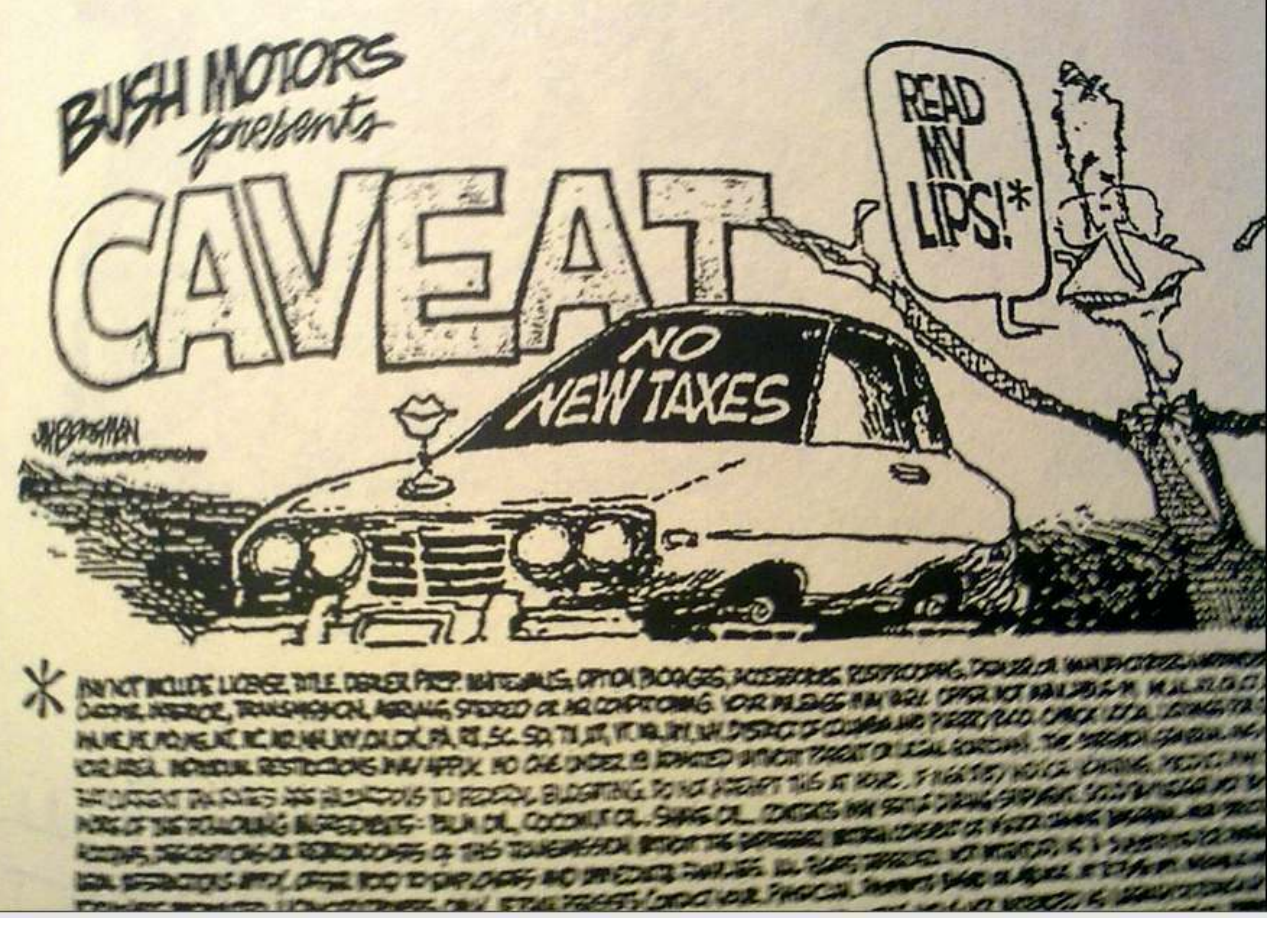

Cartoon 3

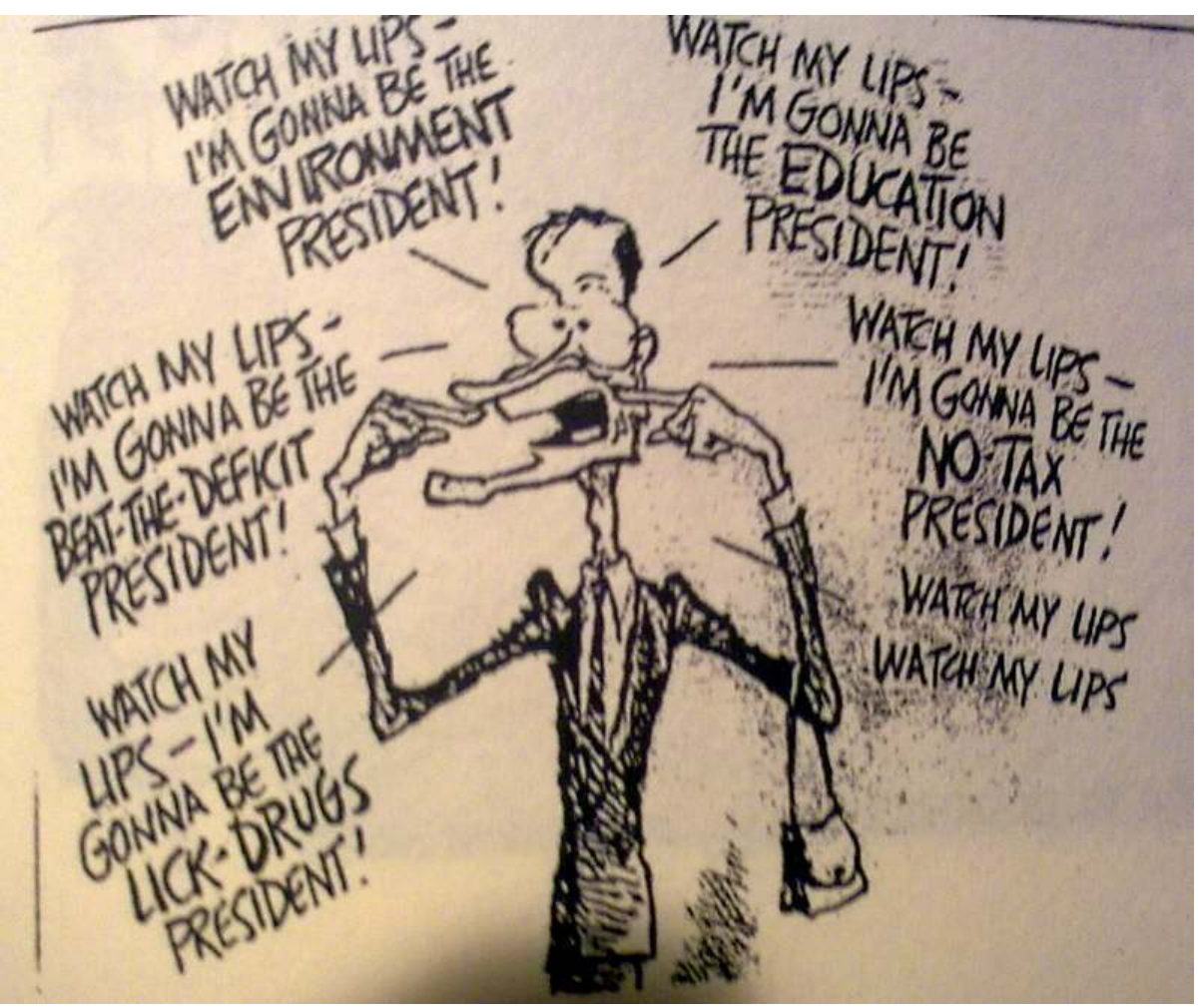


9 Les cartoons 2 et 3 se réfèrent à l'une des petites phrases les plus célèbres depuis «I like Ike " de la campagne présidentielle de 1952: il s'agit de "Read my lips, no new taxes" prononcée pour la première fois par George Bush dans son discours lors de la Convention républicaine de 1988. Répétée tout au long de sa campagne, cette phrase a été détournée pendant la campagne de 1992 pour critiquer le bilan économique de son premier mandat. Dans le cartoon de John Borgman (2), Bush est transformé en vendeur de voitures d'occasion vantant les mérites d'un véhicule douteux (si on regarde de près on remarquera l'absence de roues, par exemple). On reconnait immédiatement le style de la publicité aux apparences alléchantes: «Read my lips », suivi d'une étoile, renvoie à un paragraphe d'écriture quasi illisible (the fine print, en anglais), mais dans lequel, en fait, est regroupée une collection très hétéroclite de précautions d'emploi : "your mileage may vary" côtoie des restrictions comme "no one under 18 admitted without parent or legal guardian ", des mises en garde telles que " the Surgeon General has determined the current tax rates are hazardous to federal budgetting ", ou des instructions du genre "if ingested, induce vomiting » et « if pain persists consult your physician ». Si on arrive jusqu'aux deux dernières lignes du texte on peut lire "'Read my lips' is a registered trademark of the 'George Bush for President' campaign committee and is not intended as legally or ethically binding "! L'autre moitié de la phrase incriminée, «no new taxes ", figure sur le pare-brise du véhicule là où l'on trouverait habituellement la liste des options et le prix du produit à vendre. Le texte à gauche, dans lequel est mis en œuvre un jeu de mots entre CAVEAT (qui signifie avertissement ou mise en garde) et Cavalier, un modèle de voiture très connue, souligne ce que l'image implique déjà : il faut se méfier des «trop bonnes » affaires. Enfin, le lecteur particulièrement averti verra le renvoi implicite à l'expression latine caveat emptor qui veut dire « Let the buyer beware! ».

10 Le dessin de Pat Oliphant (3) va encore plus loin dans la critique de cette promesse électorale non tenue. On constate d'abord la transformation de « Read my lips " en « WATCH my lips » que le personnage répète sans cesse. Ce changement, qui peut, de prime abord, sembler insignifiant, comporte en réalité un message fort : le verbe READ implique la présence d'un texte à déchiffrer et la participation active du lecteur pour comprendre le sens de l'énoncé; tandis que le verbe WATCH sous-entend la simple observation, passive, du mouvement mécanique des lèvres. L'artiste diminue également la force des propos de Bush en lui donnant une attitude efféminée (le petit sac à main); le tableau ainsi brossé (image + texte) rappelle une petite fille qui cherche désespérément à se faire remarquer. Les déclarations du Président sont d'autant plus drôles qu'il s'agit de phrases extraites de différents discours réels et que Clinton a utilisé, pendant la campagne de 1992, dans les publicités négatives contre Bush. À droite du dessin, le petit pingouin qui s'appelle Puck et qui est la particularité des dessins de cet artiste commente la scène : la phrase "The Emperor has no lips " suggère, par assimilation au conte de fées The Emperor's New Clothes, que l'on ne peut pas voir ce qui n'existe pas ; la référence au conte implique en même temps que Bush, comme l'empereur, est déconnecté de la réalité et répète bêtement les déclarations préparées par ses conseillers.

11 Une autre technique intéressante utilisée par l'artiste de l'editorial cartoon consiste en le décalage entre le sens premier du terme qu'il choisit de "mettre en scène " et le sens figuré sur lequel porte le commentaire politique. Le glissement d'un sens à l'autre peut opérer dans les deux directions: soit du sens attendu vers une nouvelle utilisation du sens créateur de l'effet de surprise ; soit du sens inattendu vers le sens habituellement ou communément accepté qui éclaircit, a posteriori, le jeu de mots mis en œuvre. L'on peut 
ainsi observer dans les cartoons 4 et $5^{4}$ l'utilisation de ces deux techniques de décalage pour commenter l'affaire Lewinsky, scandale qui a défrayé la chronique pendant une bonne partie de l'année 1998. Le premier dessin (4) est particulièrement intéressant en raison de la complexité des multiples niveaux de commentaire. D'abord, le texte encadré en haut à gauche "THE SEAL OF THE PRESIDENT» est centré au-dessus de la représentation picturale du sceau officiel, image accompagnée d'un premier commentaire «THEN ». À droite, on trouve un Clinton mécontent enfermé dans une ceinture de chasteté, avec le commentaire « NOW »; ainsi, le mot « seal » ne signifie-t-il plus le sceau du pouvoir, mais le sceau de l'interdiction. Normalement le Président contrôle ; ici il est, au contraire, contrôlé manifestement contre son gré. Plus important encore, on notera que dans la partie gauche du dessin l'artiste ne montre que le sceau du Président, sans Clinton. Il aurait pu choisir, par exemple, de montrer le Président derrière son bureau sur lequel est apposé ce sceau. Cette absence fait partie du commentaire éditorial ; l'artiste ne compare pas le comportement et la réputation du Président Clinton avant et après l'affaire Lewinsky, il compare la fonction de Président avant l'arrivée de Clinton, et depuis son élection. Le passage de la dimension symbolique du mot «seal » au sens concret et réducteur crée, en fait, un effet que j'appellerai la banalisation du sacré, c'est-à-dire la transformation volontaire d'un objet significatif sur le plan symbolique ou culturel en un objet usuel, voire ridicule. C'est cet effet qui constitue l'aspect fort du commentaire de ce dessin.

On observe l'effet inverse dans le cartoon de Borgman (5). En bas du dessin, la légende "STAINS » est centrée en dessous des deux personnages, Monica Lewinsky à gauche, Kenneth Starr à droite, et sert à commenter les objets que brandit chacun d'eux. Il s'agit, pour Monica, de la célèbre robe bleue sur laquelle une expertise d'ADN a été effectuée pour prouver que la tâche (de sperme) provenait de Clinton. L'artiste propose donc dans un premier temps le sens propre (si l'on peut dire!) et réducteur du mot "stain", puisqu'il se réfère non seulement au sens concret du terme mais désigne également une tâche spécifique, celle trouvée sur la robe de Lewinsky. À droite, Kenneth Starr, le procureur nommé pour mener l'enquête de cette affaire, tient un drapeau américain, visiblement très sale, comportant l'inscription «SMEAR INVESTIGATION ». Comme dans le dessin précédent, on peut constater une superposition de messages implicites et explicites dans cette partie du cartoon réservée au commentaire éditorial : le rajout du mot «SMEAR » comme adjectif qualificatif de l'enquête de Starr annonce clairement l'opinion de l'artiste; pour lui l'étalage scabreux des détails recueillis et publiés dans la presse tout au long de cette affaire (incarné ici dans la robe à gauche) est élevé, dans le dessin, au niveau d'une attaque malveillante contre la nation américaine tout entière, dont l'image culturelle la plus forte est celle de la profanation du drapeau. Ce glissement du sens concret de "stain » vers un sens large mais très chargé en signification culturelle produit l'effet de ce que j'appellerai cette fois la sacralisation du banal, c'est-à-dire l'élévation d'un fait ou d'un objet insignifiant à un niveau hautement symbolique.

Un dernier détail du dessin souligne la très forte critique dont Kenneth Starr fait l'objet: Borgman n'avait pas vraiment besoin de mettre le mot «Starr » sur la veste du personnage, car même si le lecteur n'avait pas compris de qui il s'agissait, il aurait pu le reconnaitre par association avec la caricature de Monica Lewinsky. L'artiste attribue donc une fonction supplémentaire à ce mot; en jouant sur un AUTRE sens de «star » (cette fois écrit avec un seul $r$ ), il nous renvoie à l'image du shérif dont le badge est en forme d'étoile; ceci impliquerait culturellement que Starr n'a pas l'envergure d'un grand 
magistrat qui mène une enquête objective, mais qu'il se réduit, au contraire, au niveau d'un petit justicier chargé d'un lynchage public. Ou encore, on peut discerner un réseau de sens qui s'établit entre STARR et les rayures de la cravate que porte le personnage, qui crée implicitement l'expression "stars and stripes", collocation évoquant le drapeau américain; on comprend alors que Starr se présente en représentant privilégié des « vraies » valeurs américaines que l'artiste oppose au résultat de l'enquête, le drapeau américain sale, symbole de la perte des mêmes valeurs.

Nous allons nous tourner maintenant vers une dernière stratégie de commentaire qui dépend entièrement des connaissances culturelles de l'artiste, et par extension du lecteur, car il s'agit de l'utilisation de faits historiques pour évoquer un événement contemporain. Là aussi, comme dans la stratégie de détournement que nous venons de voir, la réactualisation d'un fait historique peut opérer de différentes manières et à différents niveaux de sens. Les cartoons 6 et 7 font appel à une connaissance culturelle assez générale ; le dessin de Borgman (6) utilise l'Inquisition pour illustrer son opinion concernant l'enquête sur l'affaire Lewinsky au moment où les parlementaires débattaient du bien-fondé de la destitution du Président. On notera ici que l'éléphant est la représentation traditionnelle, dans l'editorial cartoon, du parti républicain, et ce depuis Thomas Nast qui en est le créateur ; le lecteur comprend donc que les Républicains, au lieu de torturer Clinton jusqu'aux aveux à la manière des grands inquisiteurs espagnols, souhaitent «boucler » l'affaire au plus vite compte tenu de la côte de popularité de leur victime (message explicité dans la légende «Let's wrap this up... Clinton's approval rating just hit $105 \%$ »). L'éléphant républicain figure de nouveau dans le deuxième dessin (7) qui s'inspire de la légende de Robin des Bois : la transformation habile du nom « Robin Hood » en "Robbin' Hood» (le mot "hood» prenant ainsi la signification "voyou»), accompagnée d'un changement du "Sheriff of Nottingham" en "Sheriff of Spendingham " sert à transmettre le message de l'artiste qui commente la politique dépensière des Républicains et leurs tentatives futiles (voir l'épée en bois) pour déposséder le Président du surplus budgétaire (représenté dans le dessin par un coffre sur le toit de la diligence).

Une deuxième utilisation de faits historiques repose spécifiquement sur la mise en scène d'événements tirés de l'histoire des États-Unis. Il faut rappeler d'abord que l'histoire des États-Unis s'étale sur une période très courte comparée aux grandes civilisations européennes par exemple; de plus, dans l'histoire des États unis se mélangent assez facilement des faits historiques véritables, et des mythes ou légendes sans fondement historique réel. Tel est le cas dans le dessin intitulé « William Washington Clinton and the Cherry Tree » (8). L'artiste Jeff McNelly opère un télescopage d'une réalité historique (les propos de Clinton lors de son témoignage télévisé dans l'affaire Lewinsky «I was legally accurate when I said I didn't have sex with Ms. Lewinsky ») et d'un " mythe » historique selon lequel George Washington, alors enfant, aurait avoué à sa mère "I cannot tell a lie, it is I who chopped down the cherry tree ». La référence historique sert donc à mettre en relief le sous-texte du commentaire, c'est-à-dire que d'autres présidents (dont Washington est le modèle dans l'imaginaire culturel) étaient connus pour leurs valeurs morales irréprochables. Ce n'est pas le cas de Clinton, accusé de parjure dans cette affaire et dont la seule réponse à ces accusations fut une discussion sur l'exactitude juridique des termes utilisés. Les propos du petit Clinton dans le dessin prennent ainsi une dimension transhistorique : à cause de ses mensonges, il remet en question l'honnêteté de tous les présidents américains depuis 200 ans. 
16 L'autre cartoon de MacNelly, «Clinton's Last Stand» (9), prend son appui dans le plus grand mythe culturel de la Frontière dans l'imaginaire populaire américain, celui de Custer's Last Stand. Selon l'historien culturel Richard Slotkin, le Général Custer et la débâcle militaire dont il est responsable (la défaite des forces armées sous ses ordres à la bataille de Little Big Horn) sont passés du fait historique au mythe culturel grâce aux journalistes de l'époque qui voyaient en Custer l'incarnation des conflits socio-politiques qui déchiraient la nation dans le sillage de la Guerre de Sécession; plus encore, il incarnait les oppositions fondamentales qui font de lui un héros du mythe de la Frontière :

[...] the wartime imagery of Custer as the liminal hero, the boy-man whose sexual character is on the border between masculine adulthood and the passionate nature of woman [...] avails itself of the central characteristic of the mythic hero: his incarnation of the polar oppositions of a conflict of values or ideological imperatives. Custer is presented as the meeting point of the positive and negative forces in American culture - masculinity and femininity, adulthood and childhood, civilization and savagery, sanity and madness, order and disorder. As one who balances on a turning point between these orders and qualities, he is able to draw knowledge and power from both; but that very position makes him the embodiment of trouble and conflict, unstable and dangerous as a moral reference point. (Slotkin 1985 : 454-455)

17 Cette référence directe à tout le réseau de sens lié au personnage historique et mythique de Custer, au service ici d'une interprétation réactualisée, fait donc appel aux connaissances culturelles du lecteur.

18 Quoi qu'il en soit, dans le cartoon on voit Clinton apeuré, tout petit au fond de son fauteuil et retranché derrière son bureau dans The Oval Office; c'est en toute apparence sa dernière position de repli avant sa mise à mort médiatique (ses aveux télévisés d'août 1998). La situation est si désespérée que même l'aigle au sommet du drapeau, en haut à gauche de l'image, se cache derrière ses ailes. L'allusion à Custer's Last Stand crée une ambiguïté intéressante par le biais de laquelle l'artiste oblige le lecteur à interpréter le dessin selon ses propres critères : soit on considère que Clinton est un héros solitaire qui fait face aux hordes ennemies et représente donc un personnage historique exemplaire à cause de son courage face à l'adversité ; soit on choisit de voir Clinton comme un antihéros de l'histoire américaine, l'antithèse même des valeurs américaines qu'il est censé représenter, et qui mérite le triste sort que l'histoire lui réserve.

Every contemporary interpretation of [Custer's Last Stand], whatever its partisan purpose or ideological mission, necessarily begins by offering its own reading of Custer's character and motives, his skill as a commander and degree of culpability for the defeat. (Slotkin $1985: 477$ )

19 Ainsi Clinton incarne-t-il comme Custer, au plus haut niveau symbolique, le conflit des valeurs inhérent à l'identité américaine: l'opposition entre les forces positives et négatives du bien et du mal, du rationnel et de l'irrationnel, de l'ordre et du chaos. En réunissant ces deux personnages historiques en un seul lieu, l'artiste nous fait réfléchir aux différents sens parfois contradictoires qu'il est possible d'attribuer à un acte politique donné.

20 En fin de compte, force est de constater que l'editorial cartoon constitue une forme de discours politique extrêmement spécialisé, dans lequel les effets croisés entre image et texte fonctionnent au service d'un commentaire restant souvent dans le domaine du nondit. Et surtout, au-delà de leur capacité à nous faire rire, ils nous poussent à réfléchir sur 
tous les sens à donner aux événements politiques que l'on prend parfois trop, ou parfois pas assez, au sérieux.

\section{BIBLIOGRAPHIE}

Allwright, Dick and Kathleen Bailey. 1991. Focus on the Language Classroom. Cambridge : Cambridge University Press.

Bowen, Tim and Jonathan Marks. 1994. Inside Teaching: Options for English language teachers. Londres : Heinemann.

Brooks, Charles (dir). 1993. Best Editorial Cartoons of the Year: 1993 edition. Gretna, LA : Pelican Publishing Company.

Brooks, Charles (dir). 1999. Best Editorial Cartoons of the Year: 1999 edition. Gretna, LLA : Pelican Publishing Company.

Hess, Stephen et Sandy Northrup. 1996. Drawn and Quartered: The History of the American Political Cartoon. Montgomery, AL : Elliott \& Clark Publishing.

Hill, Draper. « Pictures at a prosecution: Thomas Nast's frustrations covering the first presidential impeachment », site Internet, The Association of American Editorial Cartoonists. < http://www.detnews.com/AAEC/winter99/history/history.html>.

Kallaugher, Kevin. 2000. "Worth a thousand words: A picture of contemporary political satire », site Internet, The Association of American Editorial Cartoonists. <http://www.detnews.com/ $\mathrm{AAEC} / \mathrm{KAL} / \mathrm{KAL} \cdot \mathrm{html}>$

Slotkin, Richard. 1985. The Fatal Environment: The Myth of the Frontier in the Age of Industrialization 1800-1890. New York : Harper Perennial.

\section{Sites Internet}

http://www.batemania.com

http://borgman.enquirer.com

http://www.grimmy.com

http://lcweb.loc.gov/exhibits/oliphant

http://macnelly.com

\section{ANNEXES}

\section{Cartoon 4}




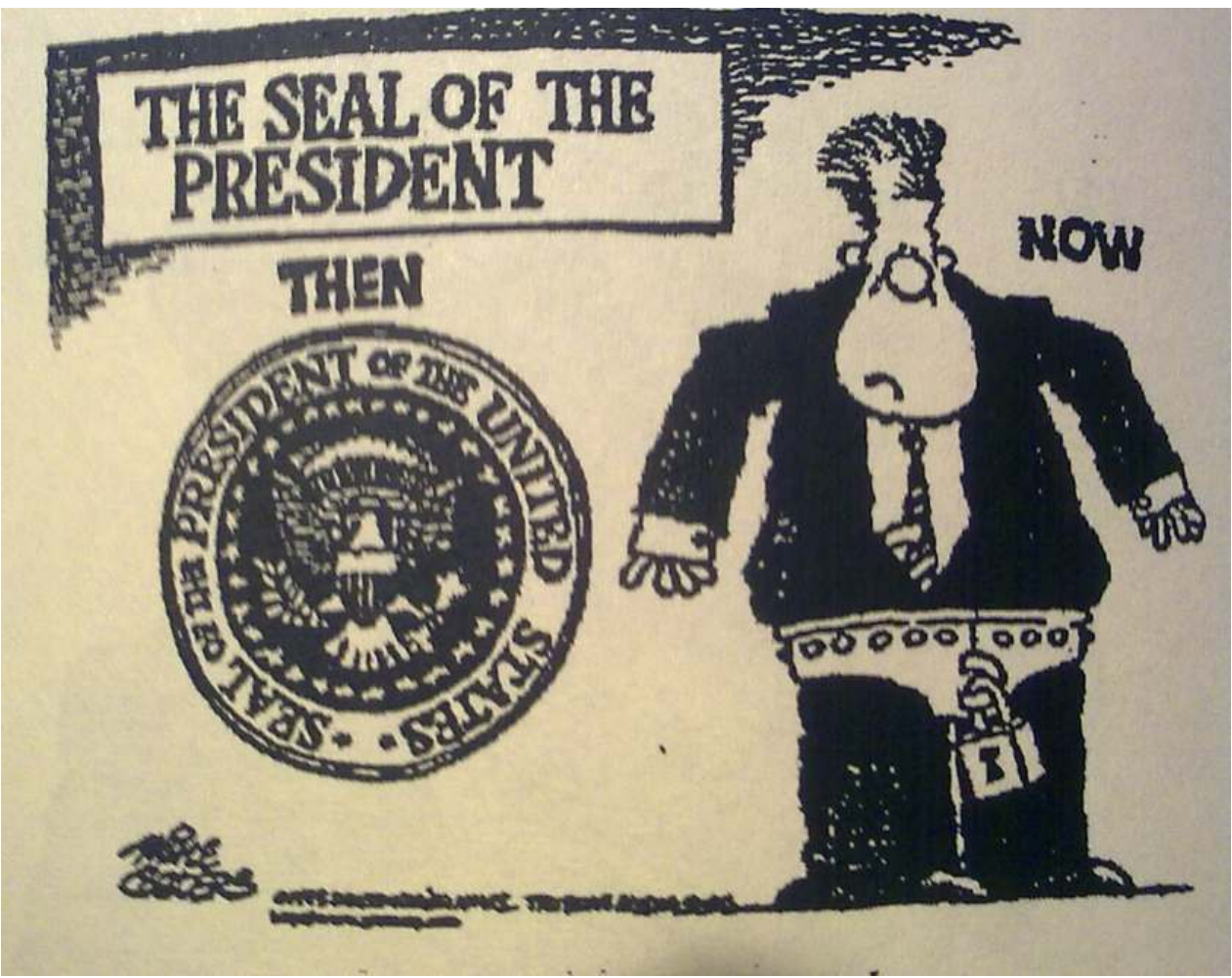

\section{Cartoon 5}

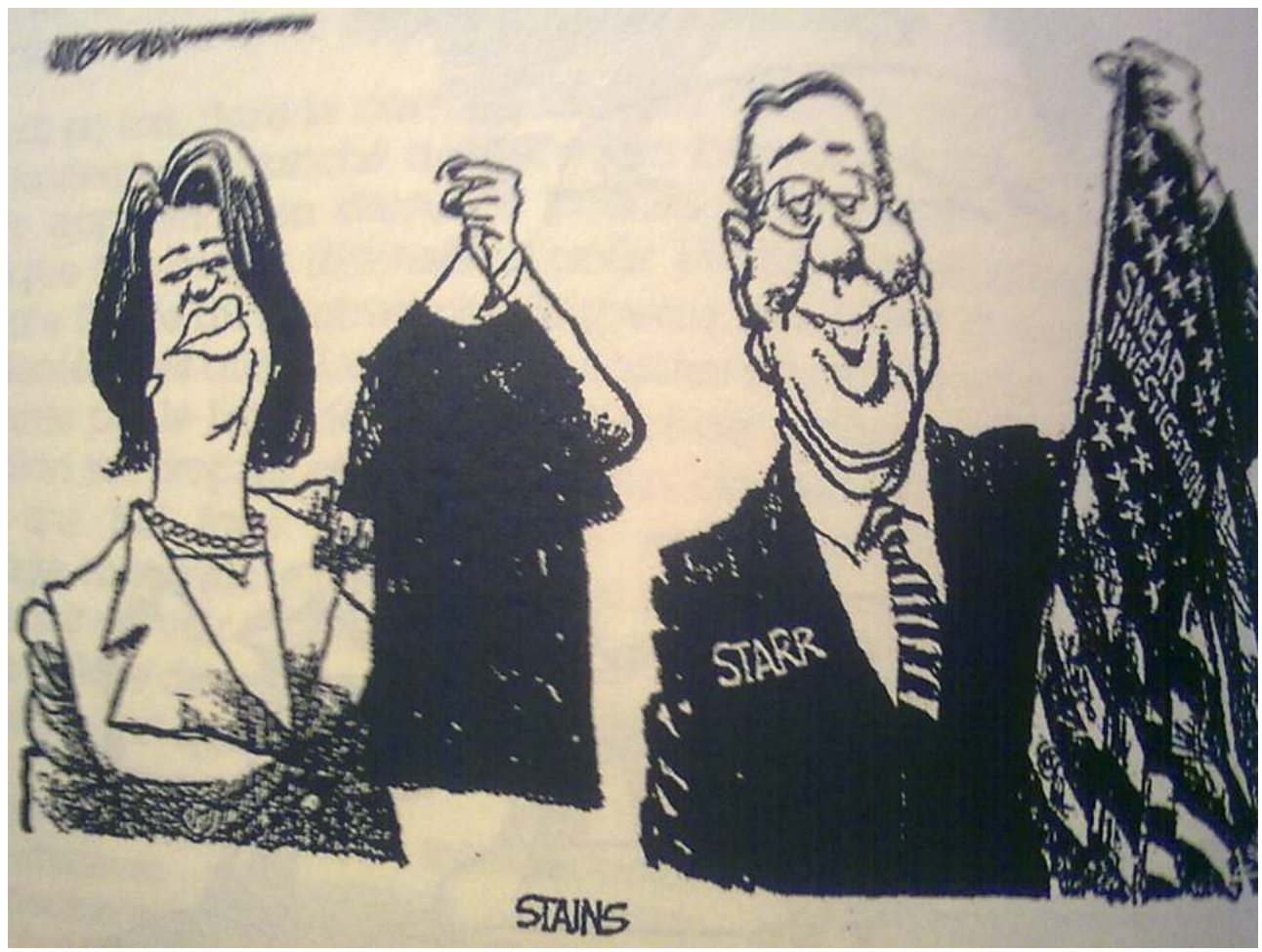

Cartoon 6 


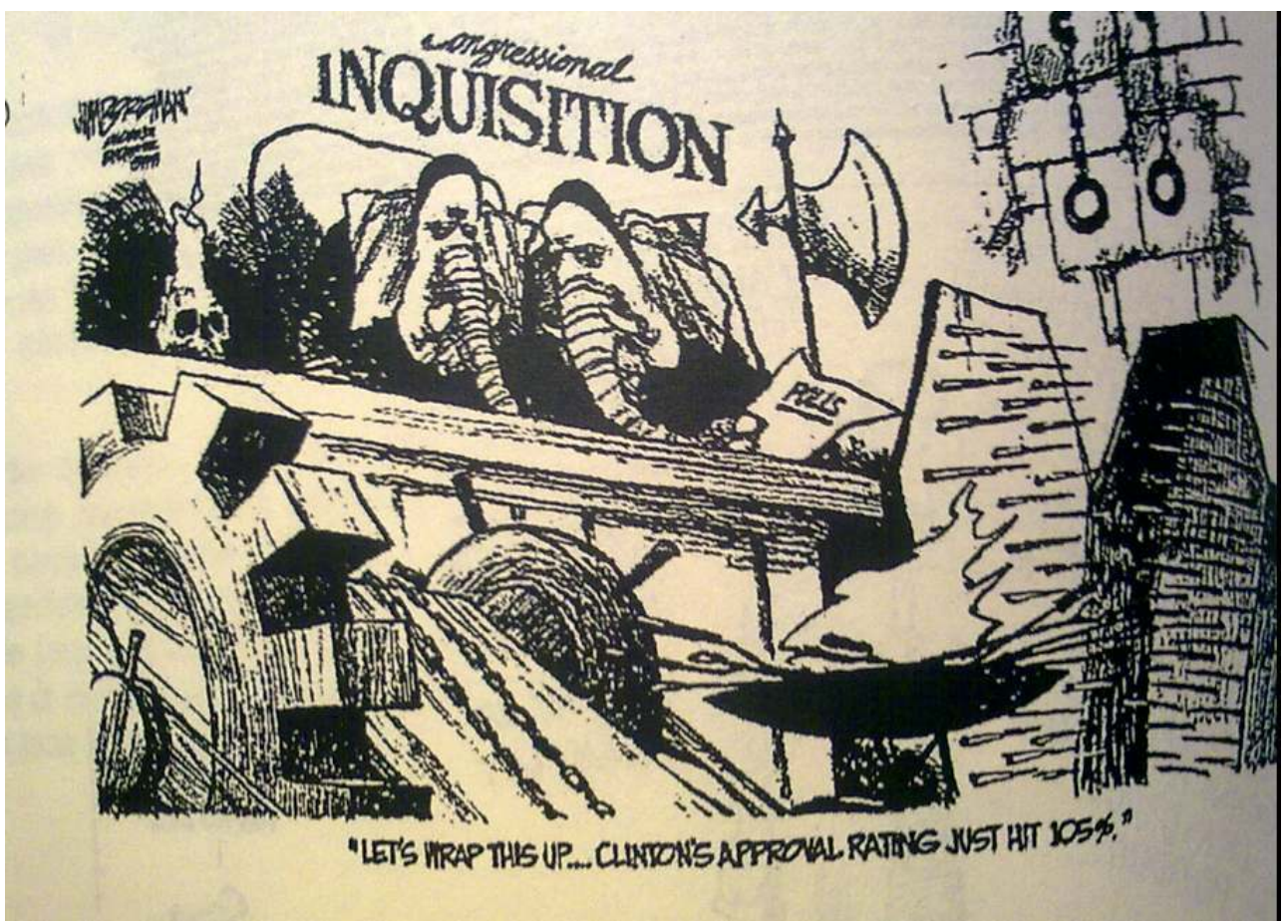

\section{Cartoon 7}

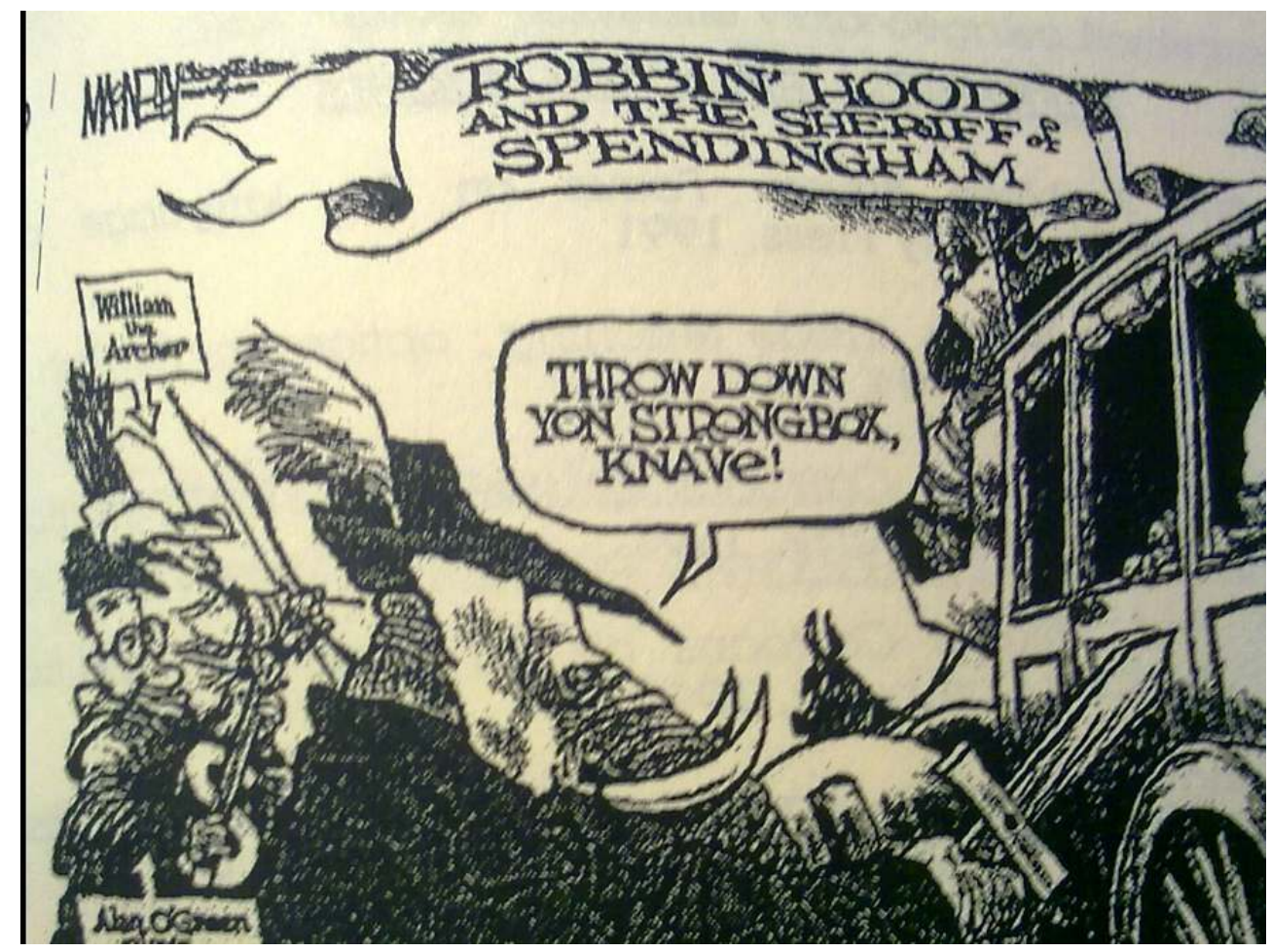

Cartoon 8 


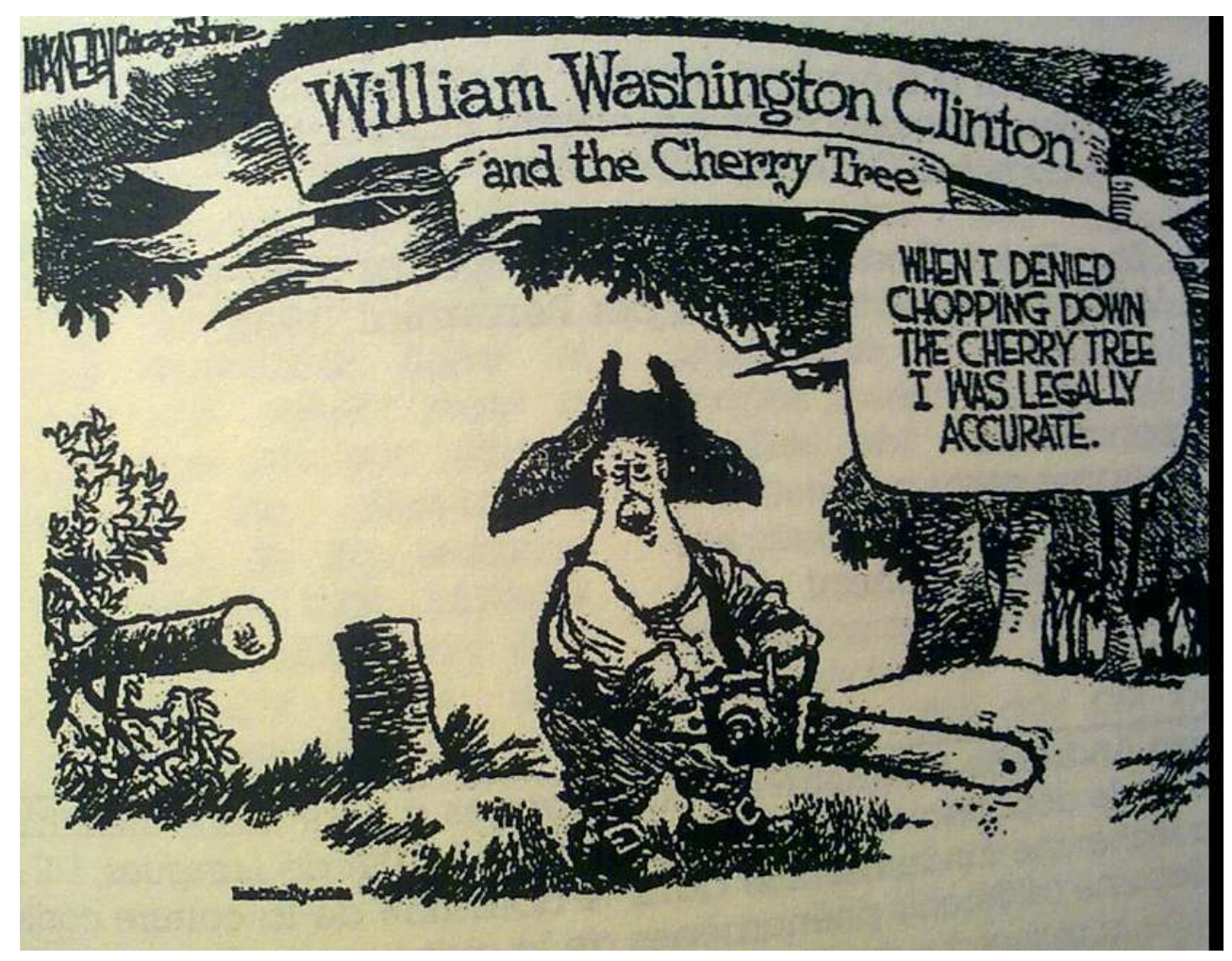

Cartoon 9

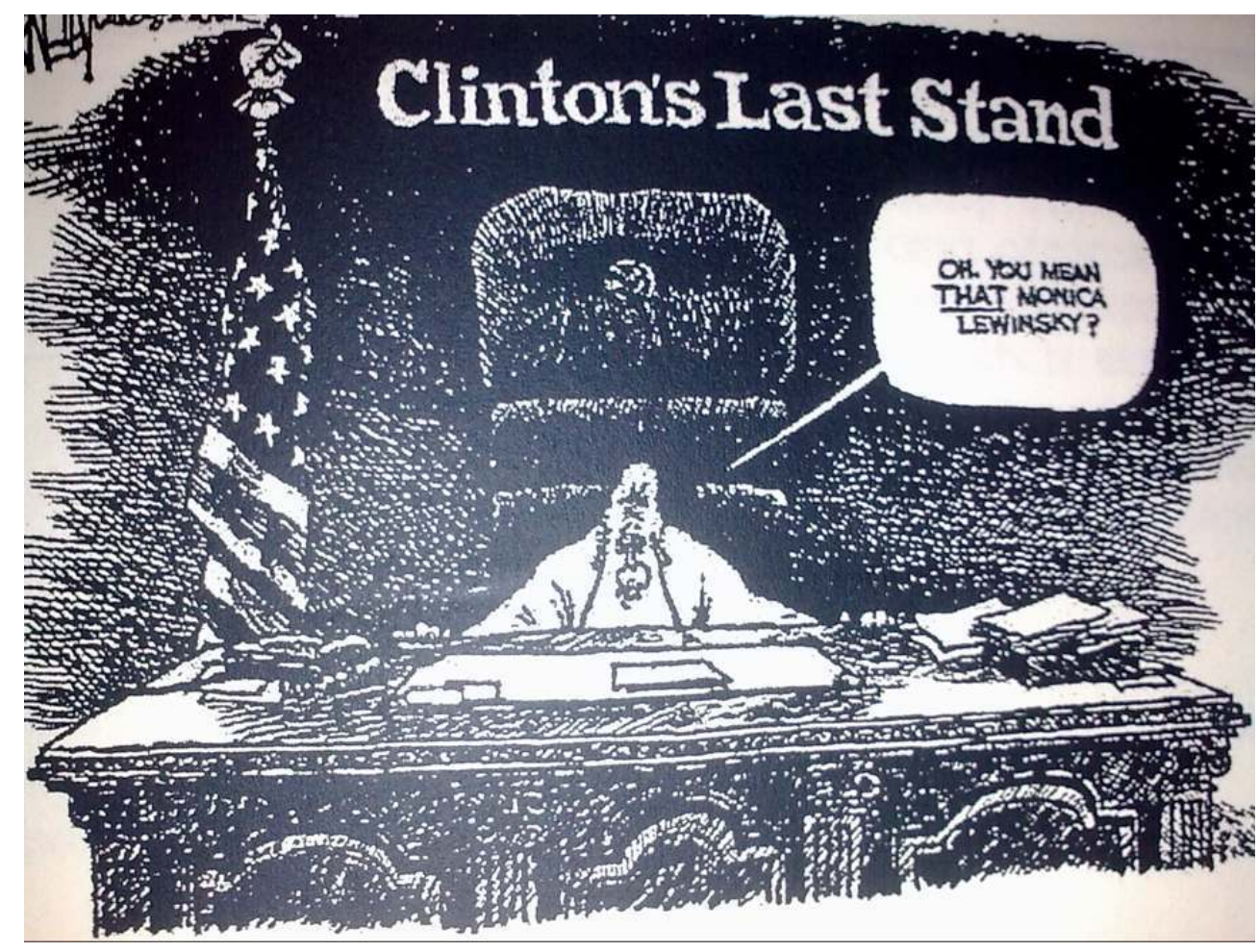




\section{NOTES}

1. Hess and Northrup (1996: 13): "In less than 10 seconds the skilled cartoonist must establish audience recognition of his or her visual symbol, make a political statement, and sell newspapers or magazines".

2. Toutes les informations relatives à l'histoire de l'editorial cartoon proviennent de Hess and Northrup, Drawn and Quartered, chapitre 1 « The Birth of a National Identity » et chapitre 3 « The Cartoon Comes of Age ».

3. Voir les références bibliographiques pour les adresses Internet de ces sites.

4. Voir en annexe.

\section{RÉSUMÉS}

Cet article propose d'analyser le rôle que jouent les editorial cartoons aux États-Unis, surtout dans la vie politique de la nation, et d'étudier les techniques mises en œuvre qui font de ces cartoons une arme puissante de commentaire éditorial. Combinaison de dessin humoristique ou caricatural et de propos soit sous forme de légende, soit sous forme de bulles qui font parler les personnages, l'editorial cartoon prend son appui dans différentes stratégies : le détournement des propos "médiatiques », le décalage entre sens propre et sens figuré, la réutilisation d'images ou de propos de l'actualité, la remise en contexte contemporain d'images ou de discours historiques... C'est par la subversion qu'amène ce jeu subtil entre l'image et le texte que l'artiste fait son commentaire, exprime son opinion, ou invite le lecteur à apprécier les sens multiples qu'il est possible d'attribuer à l'œuvre.

The purpose of this article is to analyse the role played by editorial cartoons in the United States -in particular in the political arena- and to study the techniques used by the artists which make this a powerful vehicle of political commentary. Composed of a humorous drawing and captions or speech bubbles, the editorial cartoon draws its strength from the strategic use of different forms of subversion: the "hijacking" of sound bites and slogans, the play on literal and figurative meaning, the metaphorical rendition of current events, the "updating" of historical events or discourse... It is this subtle balance between the explicit (text and image) and the implicit (the underlying message and cultural ramifications) through which the artist produces his/her commentary and invites the reader to unravel the multiple meanings that make a good editorial cartoon.

\section{INDEX}

Keywords : cartoon, image, political discourse

Mots-clés : discours politique, image, cartoon 


\section{AUTEUR}

\section{DONNA ANDRÉOLLE}

Donna Andréolle est maître de conférences à l'Université Stendhal Grenoble 3, dans le département d'Anglais appliqué (UFR de Langues, LEA). Hormis sa recherche fondamentale dans le domaine de la culture contemporaine américaine (différents phénomènes de la culture populaire depuis 1965), elle fait de la recherche appliquée dans le domaine des TICE et leurs utilisations pédagogiques dans l'enseignement des LANSAD et dans les centres d'autoformation. dandreolle@gmail.com 